\title{
Requirements of ERP Vendors using Hybrid Hierarchy Process with Artificial Neural Network (Hahp-Ann) Method
}

\author{
S.Hameed Ibrahim, S.Seetha Raman, M.Gowtham
}

\begin{abstract}
The Enterprise Resource Planning framework (ERP) has been called attention to as special data frameworks paradigm. In any case, accomplishing an appropriate degree of ERP achievement depends on an assortment of factors that are identified with an association or venture condition.Those ERP projects should be satisfied by the customers and vendors in terms of ease of accessibility, flexibility, efficiency and reliability. In our existing work, AHP-RCF method uses requirements in the rank based priority level. However, it has not been discussed about the decision criteria of the customers. In our paper, a mix approach between the AHP and ANN has been created to assess and choose the good degree of customization that is the requirements that can perform customization in a well efficient manner. The proposed method HAHP-ANN is used to measure the weight of customization and various structures of multi-layer neural networks have been analysed for the optimization. Also the learning of projectinformation gathering is done by using mix SVM classification approach based on which dynamic updation about the projectneeds can be provided to the user customization. The general valuation of the research strategy is led in the java modelbackgroundcondition from which it is demonstrated that the proposed research procedure prompts give the ideal result than the current research systems.
\end{abstract}

Key words:Hybrid Analytic Hierarchy Process-Artificial Neural Network (HAHP-ANN), Enterprise Resource Planning system (ERP), mix SVM.

\section{INTRODUCTION}

ERP is an incorporated, office endeavor wide data framework that consolidates all essential business capacities like production, purchase, stock control, deals, account, humanresource. Organizations need ERP usage with the end goal of client-order combination, regularization of production process, decrease of stock level and request planning time, institutionalization of HR data. Today organizations work in a financial situation where client requests are persistently altering and expanding. In present scenario, an incredible count of contenders are in spots and rivalry is so furious. Quality and cost don't get the job done in rivalry and consequently new challenge factors are required like deliverytime in correct period and modify item [1].These organizations endeavor to lessen whole expense through store network, supply chain cycle, and stock.

Revised Manuscript Received on September 10, 2019.

S.Hameed Ibrahim, Assistant Professor(SRG), Department of Computer Applications, Kumaraguru College of Technology, Coimbatore, Tamilnadu, India.

(Email: hameedibrahim.s.mca@kct.ac.in)

S.Seetha Raman, PG Scholar, Department of Computer Applications, Kumaraguru College of Technology, Coimbatore, Tamilnadu, India.

(Email: seetharaman.17mca@kct.ac.in)

M.Gowtham, PG Scholar, Department of Computer Applications, Kumaraguru College of Technology, Coimbatore, Tamilnadu, India.

(Email: gowtham.17mca@kct.ac.in)
Furthermore, they solicitation expanding assorted variety of item, progressively exact conveyance dates and organizing the supply and creation viably [2], [3].

The cutting edge use of the duration frequently alludes to Artificial Neural Networks, which are made out of counterfeit neurons or hubs. Neural networks give another approach to highlight extraction (utilizing hidden layers) and characterization (for example multi- layer perception). The perceptron is basically a direct classifier for ordering information indicated by parameters and a yield work. Its parameters are adjusted with an ad-hoc principle like stochastic steepest inclination plunge. Since the internal item is a direct administrator in the input space, the perception could just consummately arrange a lot of information for which various classes are straightly detachable in the info.space, while it regularly bombs totally for non-distinct information.

The general organization of the proposed research technique is given as pursues: In section 2, audit of the fluctuating related research strategies has been given. In section 3, proposed research work has been talked about in detail. In section 4 , trial assessment of the proposed research method has been given with necessity models. At last in section 5, by and large assessment of the exploration strategy is finished up with reasonable measurements.

\section{RELATED WORK}

Vaidya \& Kumar [4] exhibited a writing survey of the utilizations of AHP .It is a various criteria basic leadership device that has been utilized in practically every one of the applications related with conclusion-making. Out of various uses of AHP, this article covers a chosen few, which could be of wide enthusiasm to the analysts and experts. The article fundamentally analyses a portion of the papers distributed in global diaries of high notoriety, and gives a concise thought regarding a considerable lot of the alluded productions. The references have likewise been gathered district savvy and year-wise so as to follow the development of AHP applications.

Kumar et al., [5] exhibited a mix model utilizing AHP andNNs hypothesis to survey merchantpresentation. The model comprises of 2components: Componentonegives AHP utilizing pair savvy comparison of principles for all sellers, In this procedure the significance of the standards is additionally gotten utilizing an iterative procedure Component two uses the aftereffects of AHP to NN model 
for seller determination. The outcomes yield the best seller and proper mark to think about the presentation of every merchant. Choice of anothermerchants additionally can be completed by utilizing the authentic information. Approval of the whole created calculation has been completed independently.

Quin et al., [6] displayed Artificial neural network (ANN) and a developmental enhancement based calculation had been created. This calculation is professed to be useful for down to earth modern applications particularly for dynamic environments. A neural network has at least one input hubs and at least one neurons. Some neuron's yields are the yield of the network. The network is characterized by the neurons and their associations and loads. All neurons are sorted out into layers; the succession of layers characterizes the request where the enactments are registered.

Ibrahim et al., [7] proposed look into structure, ideal and solid undertaking asset arranging framework in particular AHP-RCF framework.. In this exploration strategy, necessities of many types in particular application prerequisite, process necessities and plan prerequisites are positioned dependent on their need level utilizing AHP technique. And furthermore, to improve the customization prerequisite and performing it in the productive manner, here client necessities are assembled in the dynamic way. Dynamic client connection is empowered to accumulate the client characterized from prerequisites at run time which would be assessed and adapted progressively utilizing SVM grouping calculation to improve the future programming necessity situation.

\section{PROPOSED METHODOLOGY}

ERP customization is an expansive term which alludes to the changes made to the ERP programming to meet the customer's necessities that are not upheld by the merchant as a standard component. Clients' necessities structure the base for ERP programming customization. In ordinary software projects, the clients indicate their prerequisites during the necessities gathering phase of the product advancement process and the product item is developed appropriately.

The proposed methodology gives a mix approach, coordinating a Analytic hierarchy process with a $0-1$ whole number advancement model, for supporting basic conclusion-making exercises inside the ANN that gives a frameworks improvement projects. The AHP segment of this screening procedure enables the decision-maker to build up a partner relationship by setting up assessment criteria , determining criteria loads and after that assessing alternative activities against the built up criteria. Incorporating the consequences of the AHP assessment exertion with a scientific programming model enables decision maker to advance the portfolio given a lot of compelled resource. To improve the venture customization a combination of closest neighbor classifier and mix SVM for learning necessities is utilized.

\subsection{Hybridized And Learning Based Decision Making} System For Project Customization

A mix approach between the Analytic Hierarchical Process and Artificial Neural Network has been created to assess and choose the best level of customization that is the prerequisites that can perform customization in the well proficient way. The mix methodology associated with the basic conclusion-making process, that keep the decisionmaker effectively drew in during the whole screening basic conclusion-making procedure is the establishment with which every single other movement cooperate. Conclusion maker must characterize the choice progression that is characterize criteria, infer criteria loads, and assess projects against the criteria. These exercises will be tended to in more detail in the accompanying segments. When the needs for each undertaking option have been resolved, they are consolidated into the portfolio streamlining part of the philosophy where they speak to esteem coefficients for a straight target work. The capacity to lead affectability analysis is planned into the philosophy and gives the conclusion maker extra understanding with respect to the heartiness of a portfolio.

\section{Analytical hierarchy process (AHP)}

An AHP is a helpful and adaptable basic conclusion making model which can be utilized for both subjective and quantitative attributes. As the attributes are resolved and the loads are processed by pair insightful examination framework, comparable system can be connected to ascertain the weights of the choices. The pair insightful examination matrix of choices is organized dependent on property. The outcome is another proportional matrix for every measure, with its comparing eigenvector. The technique is rehashed for all features, and the quantity of every option and model is obtained. Then, the score of every option is duplicated by the heaviness of the relating property. Toward the end, every one of the numbers for an option are summed up to locate the general score, and the final calculation results demonstrate the significance of every option $[8,9]$. The options are then positioned by their determined qualities. By and large, issues related with AHP are part into three sections.

Organizing the issue; assessment of local priorities; assurance of global priorities. The fundamental element of AHP is pair savvy correlations which empower decision makings to get the best choice. For all intents and purposes, AHP incorporates the accompanying advances: 1. shaping the characterized qualities progressively. 2. Creating judgment lattice utilizing pair savvy correlation. 3. Registering a need vector to weight segments of the lattice. 4. Deciding global priorities by gathering all local priorities with the use of a simple weighted whole. 5. Toward the end, the eigen value is achieved to assess the quality of the steadiness proportion of the relative framework and distinguish whether to acknowledge the data.

\subsubsection{Artificial neural network}

An ANN is an component that can obtain, speak to, and figure a guide of data starting with one multivariate space then onto the next utilizing an informational index speaking to that mapping [10]. The reason for an ANN is to assemble a model of the information creating process with the goal that the system can sum up and anticipate yields from data 
sources. The multi-layer perceptron (MLP) neural networks, which has been depicted is one of the most generally utilized ANNs. The MLP comprises of three layers (hidden, output, and input) and can distinguish connections that are non-linear in nature. The MLP networks are prepared by error-correction realizing, which implies that the ideal reaction of the framework must be known; a backpropogation (BP) calculation should likewise be known. The $\mathrm{S}$-formed sigmoid capacity is normally utilized as the exchange work.

The BP calculation arbitrarily chooses the underlying loads. At that point, the distinction between the normal and determined yield esteems over all perceptions is abridged utilizing the mean-square error. After every one of the perceptions are exhibited to the system, the loads are altered by a summed up delta rule [11]. This procedure of bolstering forward sign and back-propagating errors is rehashed iteratively until the error balances out at a low level [12].The working standard of NN is given as pursues:

1 The sources of info (for example $S_{1}, S_{2}, \ldots, S_{1}$ ) are the movement of gathering information from the applicable resources.

2 The weightsresist the impacts of the contributions on the neuron. Otherwise, an ANN spares its data over its connections and every connection has a weight (for example $\left.\mathrm{W}_{1}, \mathrm{~W}_{2}, \ldots, \mathrm{W}_{1}\right)$. These loads are always differed while attempting to improve the connection in the middle of the data sources and yields. Synaptic loads portray themselves with their quality (esteem) which compares to the significance of the data originating from every neuron. Then, the data is encoded in these quality loads.

3 Total capacity is to ascertain the net information readings from the handling components. (for example sum = $\left.\mathrm{W}_{1} \mathrm{~S}_{1}+\mathrm{W}_{2} \mathrm{~S}_{2}+\mathrm{W}_{3} \mathrm{~S}_{3}+\ldots+\mathrm{W}_{1} \mathrm{~S}_{1}\right)$.

4 Transfer (enactment) work (f) decides the yield of the neuron by tolerating the remaining info ( $\operatorname{sum}=\mathrm{W}_{1} \mathrm{~S}_{1}+$ $\mathrm{W}_{2} \mathrm{~S}_{2}+\mathrm{W}_{3} \mathrm{~S}_{3}+\ldots+\mathrm{W}_{1} \mathrm{~S}_{1}$ ) given by the summation work.

5 Outputs acknowledge the consequences of the exchange capacity and present them either to the significant preparing component or to the outside of the system.

6 Each information has its very personal load in addition to there is an extra weight called bias (b).

Preparing a NN model basically means choosing one model from the prearrangement of allowable that limits the cost model. There are various measurements of calculations accessible for preparing NN models; the vast majority of them can be seen as a direct consumption of reformation hypothesis and valuation. The greater part of the calculations utilized in preparing ANNs are utilizing some type of inclination plummet. This is finished by basically enchanting the subservient of the cost capacity as for the system parameters and afterward changing those factors in a gradient-related direction.

3.2 Hybrid Analytic Hierarchy Process-Artificial Neural Network (HAHP-Ann)

The theoretical model for seller determination utilizing mix AHP and, ANN idea is appeared in Fig. 1. As referenced previously, the mix model using analytic hierarchy process and artificial neural networks hypothesis can really survey ERP merchant execution. As referenced before the strategy, utilized here comprises of two modules. Module one applies HAHP and pair astute correlation of criteria and ERP merchants regarding every measure to acquire the weight. Module two uses the outcomes (loads) of HAHP into ANN model for ERP merchant determination. The outcomes yield the best merchant and fitting loads to know the presentation of each ERP seller. The principle calculationsare demonstrated as follows.

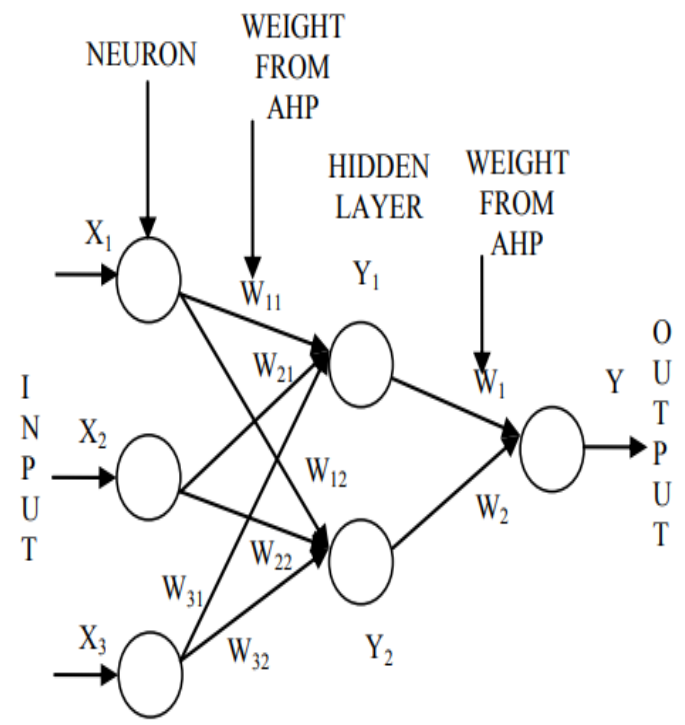

Fig. 1. Architecture of neural network

\subsubsection{Algorithm of HAHP-ANN}

\section{The algorithm is depicted below:}

Givethe input as number ofstandards chose.

Give the number of ERP merchants.

Characterize the standards utilizing Saaty's basic scale.

Feed the information of every ERP merchant.

Produce a framework for correlation of every basis of objective.

Make a framework of the estimation of loads on goal by utilizing the accompanying equation:

Weight of given standards $=$ Value of given standards/Sum of sector esteem

Create the correlation network for ERP merchant regarding given criteria.

Make next network for the computation of weight of ERP seller as for criteria by utilizing the accompanying recipe: Weight of given seller with respect to standard $=$ Value of given ERP merchant/Sum of sector esteem

Rehash the means for merchants until the standard $\mathrm{I}=0$

Make a framework for concealed cover by utilizing the accompanying equation:

Yield an incentive for concealed layer Yli $=1 /(1+\mathrm{e}$ $\alpha(\Sigma$ SiWli $)$ )

Make a grid for yield layer by utilizing following recipe: Incentive for yield layer Yvi $=1 /(1+\mathrm{e}-\alpha(\Sigma$ YliWvi $))$

Yli $=$ Total score of seller

Select the vendor of maximum. score from the above network for the best ERP vendor.

Stop. 
A mix approaches between the Analytic Hierarchical Process and Artificial Neural Network have been created to assess and choose the best level of customization that is the prerequisites that can perform customization in a well effective way.

In view of the consequences of poll, HAHP had been created. The procedure will be through a couple shrewd correlation with decide the neighborhood loads of every foundation. Ordinary system in HAHP had been pursued as needs be.

At that point ANN systems are built up that comprise of fourteen diverse preparing calculations and twelve unique setups for each preparation calculation. Thirty five data sources and one yield are utilized. During preparing, estimation of Mean Square Error for each system is resolved, and the most reduced estimation of Mean Square Error shows the best preparing presentation. For testing process, results from HAHP are utilized to as testing information. Simply the best system inside each preparation calculation had been tried, and their exhibition was estimated with coefficient of assurance (B). System that has $\mathrm{B} \geq 0.900$ is viewed as the best system. Forecast of best provider by the ANN is contrasted with the outcome determined from HAHP.

\subsection{Learning Requirements Using Mix Support Vector} Machine

This work proposes a coordination of closest neighbor classifier and support vector machine for learning necessities which can be utilized for the venture customization. With the goal that the mix SVM Classifiers are normally advanced dependent on some type of risk minimization. Exact risk minimization is one of the most normally utilized procedure where the objective is to discover a parameter setting that limits the risk:

$$
R_{\text {emp }}(\propto)=\frac{1}{2 l} \sum_{i=1}^{l}\left|y_{i}-f\left(x_{i}, \propto\right)\right|
$$

where $\propto$ is the arrangement of movable parameters and $y_{i}, x_{i}$ are the normal yield and given information, individually. Be that as it may, limiting R_emp does not really suggest the most ideal classifier.

The intensity of Hybrid SVM lies in their capacity to change information to a high dimensional space where the expectation information can be isolated utilizing a straight hyperplane. The streamlining procedure for Hybrid SVM adapting along these lines starts with the meaning of a practical that should be enhanced as far as the parameters of a hyperplane. The utilitarian is characterized with the end goal that it ensures great order (if not impeccable characterization) on the preparation information and furthermore amplifies the margin. The focuses that lie on the hyperplane fulfill,

$\mathrm{v} \cdot \mathrm{x}+\mathrm{b}=0$

wherev is the ordinary to the hyperplane and $b$ is the inclination of the hyperplane from the birthplace. Let the $\mathrm{N}$ preparing models be spoken to as tuples $\left\{x_{i}, y_{i}\right\}, \mathrm{i}=1, \ldots, \mathrm{n}$ where $y= \pm 1$ are the class lables. They fulfill the accompanying requirements,

$$
y_{i}\left(x_{i} \cdot v+b\right)-1 \geq 0 \quad \forall i
$$

The separation between the edges can be demonstrated to be $2 /\|v\|$. The objective of the improvement procedure ought to be to amplify the margin. Representing this as a quadratic enhancement issue has a few favorable circumstances and the practical can be minimally composed as,

$$
L_{p}=\frac{1}{2}\|v\|^{2}-\sum_{i=1}^{N} \alpha_{i} y_{i}\left(x_{i} \cdot v+b\right)+\sum_{i=1}^{N} \alpha_{i}
$$

Note that the quantity of errors can be described by $\sum_{i} \xi_{i}$.

We currently need to address the requirement for learning classifiers that characterize non-direct choice regions.

The proposed technique is to actualize the framework that can guarantee the presentation, ideal and adaptable undertaking resource planning system.

\section{EXPERIMENTAL RESULTS}

In this area, numerical assessment of the proposed research approach is done as far as different execution measures to break down the presentation enhancement of the projected and presentresearch methodologies. The java softwareis utilized to execute the proposed research technique. The presentation estimates considered in this work are recorded as follows:"Specificity, and Precision,Accuracy, Sensitivity". The examination is made between the proposed strategy Hybrid Analytic Hierarchy Process-Artificial Neural Network (HAHP-ANN) with the current strategies that are ANN, DC-ERPP and AHP-RCP.

Accuracy: It is characterized as level of right recognizable proof of prerequisites that can be used for project customization. That is less false positive rate. The precision of the proposed framework ought to be more than the other existing procedures, for example, DC-ERPP, ANN and AHP-RCF. The exactness worth is determined as far as the undertaking customization framework's actual positive, false positive, true negative and false negative qualities. The accuracy is determined as like as pursues:

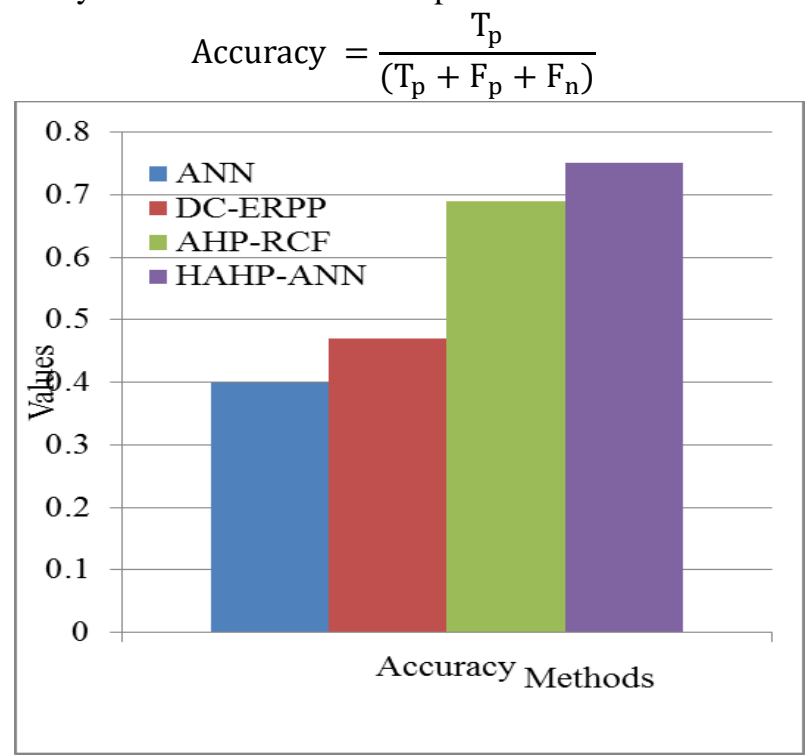

Figure 2. Accuracy comparison

The figure 2 outlines the exactness metric examination graphical portrayal. This diagrams demonstrates that the proposed research technique is superior to the current research strategies. HAHP-ANN is $6 \%$ superior to AHP$\mathrm{RCF}$, at that point $28 \%$ superior to DC-ERPP, and $35 \%$ superior to ANN.

Published By: 
Sensitivity: It is likewise called as evident positive rate which is characterized as level of accurately grouping venture customization necessities as positive. Numerically, this can be communicated as:

Sensitivity $=\frac{\text { number of true positives }}{\text { Number of true positives }+ \text { Number of false negatives }}$

The figure 3 outlines the sensitivity metric correlation graphical portrayal. This charts demonstrates that the proposed research strategy is superior to the current research strategies. HAHP-ANN is half superior to AHP-RCF, at that point $80 \%$ superior to DC-ERPP, $84.1 \%$ superior to ANN.

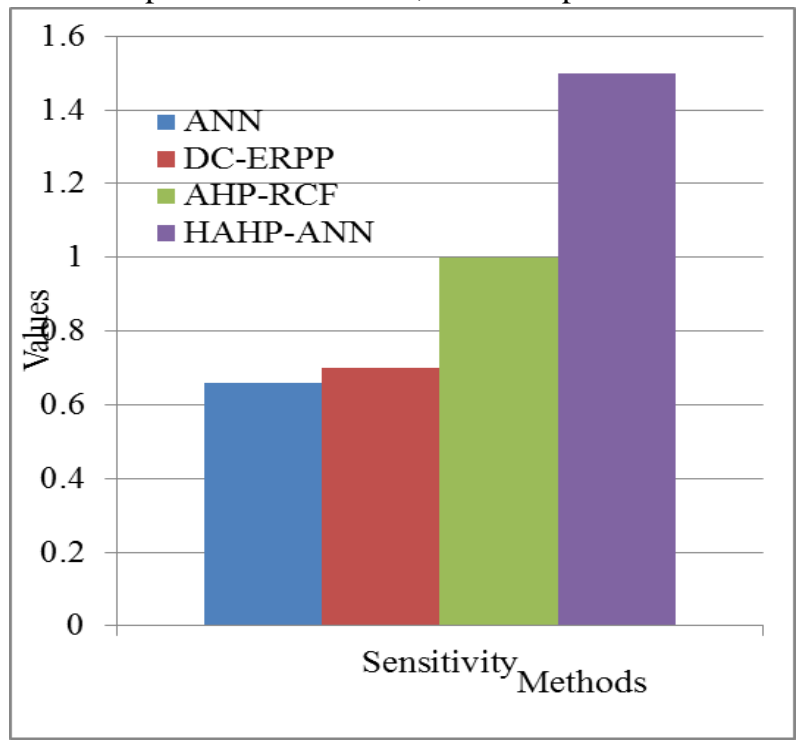

Figure 3. Sensitivity comparison

Specificity: It is additionally called as evident negative rate which is characterized as level of effectively arranging not reasonable task customization prerequisites. Scientifically, this can likewise be composed as:

Specificity $=\frac{\text { number of true negatives }}{\text { Number of true negatives }+ \text { Number of false positives }}$

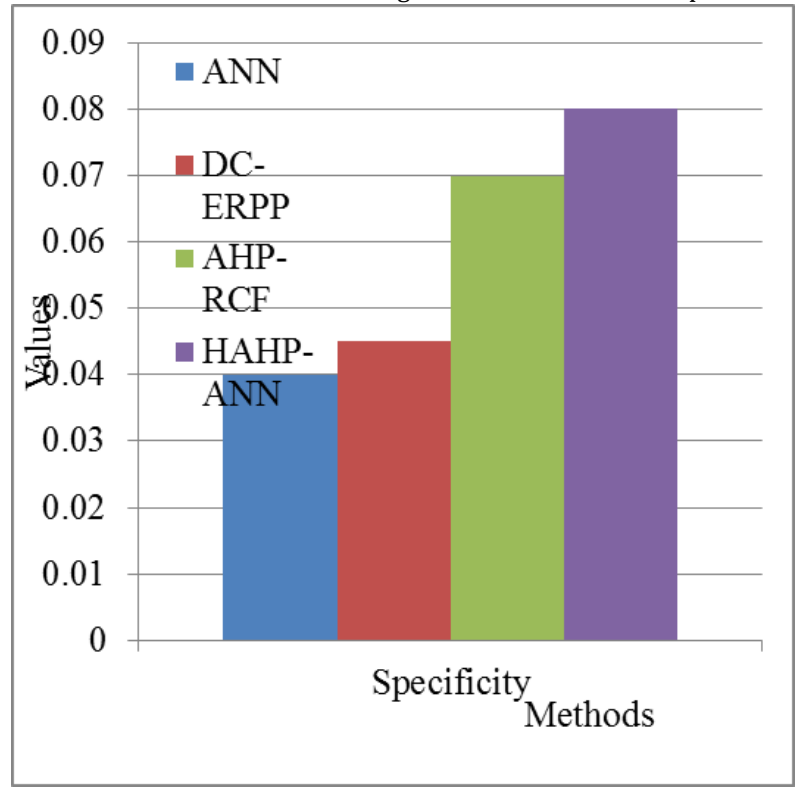

Figure 4. Specificity comparison

The figure 4 delineates the explicitness metric examination graphical portrayal. This charts demonstrates that the proposed research technique is superior to the current research strategies. HAHP-ANN is $10 \%$ superior to
AHP-RCF , then $35 \%$ is superior to DC-ERPP, and $40 \%$ superior to ANN.

Precision: It is the portion of recovered cases that are important

Precision $=\frac{\mid\{\text { relevant documents }\} \cap\{\text { retrieved documents }\} \mid}{\mid\{\text { retrieved documents }\} \mid}$

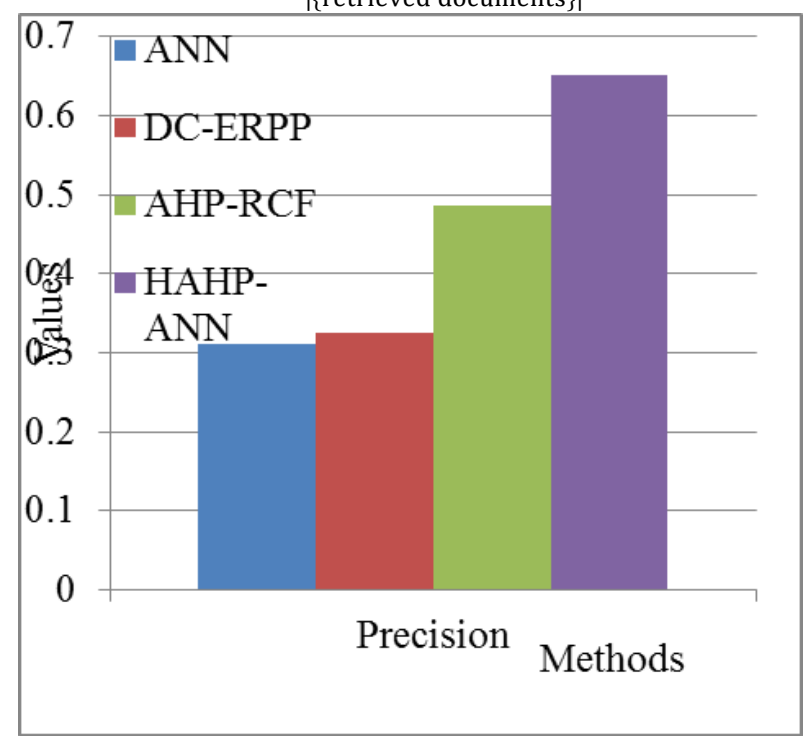

Figure 5. Precision comparison

The figure 5 delineates the accuracy metric correlation graphical portrayal. This diagrams demonstrates that the proposed research technique is superior to the current research strategies. HAHP-ANN is $16.6 \%$ superior to AHP$\mathrm{RCF}$, at that point $32.7 \%$ is superior to DC-ERPP, and $34.2 \%$ superior to $\mathrm{ANN}$.

\section{CONCLUSION}

In this work, the product advancement stage is to execute the framework that can guarantee the exhibition, ideal and adaptable endeavor asset arranging framework specifically Hybrid Analytic Hierarchy Process-Artificial Neural Network (HAHP-ANN) strategy. In the proposed technique, the mix approach with the association of the decision-maker at the pecking order of the procedure is to get the best enhancement. And furthermore, to improve the task customization, an incorporation of closest neighbor classifier and mix SVM for learning necessities is utilized. The general assessment of the examination strategy is led in the java simulation environment which it is demonstrated that the proposed research system prompts give the ideal result than the current research approaches.

\section{REFERENCES}

1. Yusuf, Y., Gunasekaran, A., \& Wu, C. (2006) Implementation of enterprise resource planning in China Technovation.

2. Liao, X., Li, Y., \& Lu, B. (2007). A model for selecting an ERP system based on linguistic information processing. Information Systems, 32, 1005-1017.

3. Xiuwu, L., Yuan, Li., \& Bing, Lu. (2007). A model for selecting an ERP system based on linguistic information processing. Information System, 32(7), 1005-1017. 
4. Vaidya, O. S., \& Kumar, S. (2006). Analytic hierarchy process: An overview of applications. European Journal of operational research, 169(1), 1-29.

5. Kumar, J., \& Roy, N. (2010). A hybrid method for vendor selection using neural network. International Journal of Computer Applications, 11(12), 35-40.

6. Quin, L. 2009. An ANN pruning algorithm based approach to vendor selection. Kybernetes, vol. 38, 3-4, pp. 314-320.

7. Balakrishnan, Nagaraj, and K. Nisi. "A deep analysis on optimization techniques for appropriate PID tuning to incline efficient artificial pancreas." Neural Computing and Applications (2018): 1-10.

8. Ibrahim, S. H., Duraisamy, S., \&Sridevi, U. K. (2018). Flexible and reliable ERP project customization framework to improve user satisfaction level. Cluster Computing, 1-7.

9. Oztaysi B (2014) A decision model for information technology selection using AHP integrated TOPSISGrey: the case of content management systems. Knowl Based Syst 70:44-54

10. Deng X, Hu Y, Deng Y, Mahadevan S (2014) Supplier selection using AHP methodology extended by D numbers. Expert SystAppl 41:156-167

11. Saaty, T. L. 1980. The analytic hierarchy process. New York: McGraw-Hill.

12. A. D. Henriksen and A. J. Traynor, "A practical R\&D project-selection scoring tool," IEEE Trans. Eng. Manage., vol. 46, pp. 158-170, May 1999

13. Garrett J (1994) Where and shy artificial neural networks are applicable in civil engineering. J Comput Civil Eng 8:129-130 